\title{
Deposition and transport of graphene oxide in saturated and unsaturated porous media
}

Lin Liu, Bin Gao, Lei Wu, Verónica L. Morales, Liuyan Yang, Zuhao Zhou, Hao Wang

NOTICE: this is the author's version of a work that was accepted for publication in Chemical Engineering Journal. Changes resulting from the publishing process, such as peer review, editing, corrections, structural formatting, and other quality control mechanisms may not be reflected in this document. Changes may have been made to this work since it was submitted for publication. A definitive version was subsequently published in Chemical Engineering Journal, [Vol. 229, (2013)] DOI http://dx.doi.org/10.1016/j.cej.2013.06.030 


\section{Deposition and transport of graphene oxide in saturated and unsaturated porous media}

Lin $\mathrm{Liu}^{1,2,5}$, Bin $\mathrm{Gao}^{2 *}$, Lei Wu ${ }^{2}$, Verónica L. Morales ${ }^{3}$, Liuyan Yang ${ }^{4}$, Zuhao Zhou ${ }^{5}$, Hao Wang $^{5}$

1. College of Environmental Science and Engineering, Donghua University, Shanghai 201620, China

2. Department of Agricultural and Biological Engineering, University of Florida, Gainesville, FL 32611

3. SIMBIOS Centre, University of Abertay, Dundee, DDI U.K.

4. State Key Laboratory of Pollution Control and Resource Reuse, School of the Environment, Nanjing University, Nanjing 210093, China

5. State Key Laboratory of Simulation and Regulation of Water Cycle in River Basin, China Institute of Water Resources and Hydropower Research (IWHR), Beijing 100038, China

*Corresponding author, phone: (352) 392-1864 ext. 285, Fax: (352) 392-4092, email:

bg55@ufl.edu 


\section{Abstract}

In this work, sand and bubble column experiments were conducted to explore the deposition mechanisms of graphene oxide (GO) particles in porous media with various combinations of moisture content and ionic strength. Sand column experimental results indicated that retention and transport of GO in porous media were strongly dependent on solution ionic strength.

Particularly, GO showed high mobility under low ionic strength conditions in both saturated and unsaturated porous media. Increasing ionic strength dramatically increased the retention of GO particles in porous media, mainly through secondary-minimum deposition as indicated in the XDLVO interaction energy profiles. Recovery rates of GO in unsaturated sand columns were lower than that in saturated columns under the same ionic strength conditions, suggesting moisture content also played an important role in the retention of GO in porous media. Findings from the bubble column experiments showed that the GO did not attach to the air-water interface, which is consistent with the XDLVO predictions. Additional retention mechanisms, such as film straining, thus could be responsible to the reduced mobility of GO in unsaturated porous media. The experimental data of GO transport through saturated and unsaturated porous media could be accurately simulated by an advection-dispersion-reaction model.

Keywords: graphene oxide, porous media, surface deposition, air-water interface, transport modeling 


\section{Introduction}

Graphene oxide (GO) is a graphene-based and water-soluble nanomaterial, contains a range of reactive oxygen surface functional groups, and is used in various industries for its exceptional physiochemical properties (e.g., mechanical strength, electrical and thermal conductivities, etc.) [1-6]. Given the wide-ranging applications and rapid growth in production for this material, it is conceivable to expect a considerable amount of GO particles to be released into the environment [7].

Considering that Graphene nanosheets and carbon nanotubes (CNT) share many unique properties, it is not surprising that studies have identified toxic effects from both materials on a variety of mammalian organism, human and bacterial cells [8-10]. The oxygen surface functional groups make GO dispersing in water easily [11], thereby potentially increasing its environmental contamination risks when released into soil and groundwater. Because of the reactive nature of the surface functional groups and the extremely high surface area to mass ratio of the GO, the presence of mobile GO particles in soil and groundwater may alter the fate and transport of chemical compounds including hazardous materials $[12,13]$. Therefore it is import to have thorough investigations about fate and transport of GO in porous media.

Only few studies have focused on understanding the environmental fate and transport of GO. A recent study by Feriancikova and $\mathrm{Xu}$ [14] was concerned with the transport behavior of GO within saturated porous media under different ionic strength conditions and concluded that GO particles have a high mobility in saturated porous media such as sandy groundwater aquifers. To 
the authors' knowledge, no research has been conducted to examine the transport behavior of GO in unsaturated systems. It is expected that the transport behavior of GO could be more complicated in unsaturated porous media than in saturated media because of the presence of an additional phase (air) and its interfaces [15-17]. Nevertheless, a good understanding of the fate and transport of GO particles in unsaturated porous media is very much needed because it is important to assess the effectiveness of the soil vadose zone as a barrier to prevent the migration of these nanoparticles into groundwater aquifers.

The overarching objective of this work was to explore the mechanisms governing the deposition and transport of GO in porous media, particularly under unsaturated conditions. Specific objectives were to: (1) examine the stability of GO in aqueous solutions under different electrolyte conditions; (2) determine the combined effect of moisture content and solution ionic strength on the retention and transport of GO in porous media; (3) examine the interactions between GO and air-water interfaces; and 4) model the interaction and transport of the GO in saturated and unsaturated porous media. To achieve these specific objectives, laboratory experiments were conducted to study the deposition and transport of GO in saturated and unsaturated porous media. Laboratory columns packed with quartz sand at different moisture content and solution ionic strength combinations were used in the experiments. To further understand retention mechanisms unique to unsaturated systems, bubble column experiments were used to assess the interaction between the GO and air-water interface. Finally, mathematical models were applied to interpret the experimental data. 


\section{Materials and Methods}

\section{$2.1 \mathrm{GO}$}

Single layer graphene oxide (ACS Material, Medford, MA), prepared by modified hummer's method $[18,19]$, was used as received from the manufacturer. Transmission electron microscope (TEM), as measured by the manufacturer, demonstrated that the diameter range of GO particles was 1-5 $\mu \mathrm{m}$ with a thickness range of 0.8-1.2 $\mathrm{nm}$. The GO suspensions were prepared by mixing $12 \mathrm{mg}$ of pristine GO with $1000 \mathrm{~mL}$ electrolyte solutions of 1, 10 and $100 \mathrm{mM} \mathrm{NaCl}$ (i.e., three ionic strength conditions). Subsequently, the suspensions were sonicated with Misonix S3000 ultrasonicator (QSonica, Newtown, CT) for two hours to ensure thorough dispersion.

Concentrations of the dispersed GO suspensions were measured with light absorption at a wavelength of $230 \mathrm{~nm}$ with an Evolution $60 \mathrm{UV}-\mathrm{Vis}$ Spectrophotometer (Thermo Scientific, Waltham, MA) (Figure S1, supporting information). Stability of the GO suspensions in the three electrolyte solutions was also determined by monitoring the light absorption of the suspension over time for $7 \mathrm{hrs}$. Electrophoretic mobility (EPM) and the zeta potential of the GO were measured using a ZetaPlus (Brookhaven Instrument Co., Holtsville, NY).

\subsection{Porous media}

The porous medium used was quartz sand (Standard Sand \& Silica Co.) with a size range of $0.5-0.6 \mathrm{~mm}$. The sand was washed sequentially by tap water, $10 \%$ nitric acid (v:v) and deionized 
water to remove metal oxides from the surface following the cleaning procedures of Tian et al.

[20]. The zeta potential of the sand porous media under different solution chemistry conditions was measured following the method developed by Johnson et al [21].

\subsection{Bubble column experiment}

Bubble column experiments were used to examine the interaction between the GO particles and the air-water interface, following the protocol of previous studies [22, 23]. An acrylic column of $2.5 \mathrm{~cm}$ diameter and $65 \mathrm{~cm}$ height was used and the column was filled with the GO suspensions during the experiments. The bottom of the bubble column was connected to a small empty chamber, which was sealed with two stainless steel screens (50 $\mu \mathrm{m}$ mesh size), for generating air bubbles. A peristaltic pump was used to force air flow $\left(5 \mathrm{~mL} \mathrm{~min}^{-1}\right)$ into the chamber and then through the steel screens at the bottom of the column. The average radius of the air bubbles produced with this method was around $5^{*} 10^{-5} \mathrm{~m}$. Previous study indicated that this method can create uniform micro air bubbles moving from the bottom to the top within the column [23]. Additionally, four sampling ports sealed with stoppers were evenly distributed along the length of the column to sample for GO solution and determine its temporal and spatial distribution during the experiments.

\subsection{Transport of GO in porous media}

An acrylic column of $2.5 \mathrm{~cm}$ in diameter and $16.5 \mathrm{~cm}$ in height was used. The column has six 
vent holes drilled on opposite sides at $3,7.5$, and $12 \mathrm{~cm}$ from the top to the bottom. The vent holes were sealed with gas-permeable porous PTFE membranes (Milliseal Disk, Millipore) to allow air to enter the column and maintain unsaturated conditions. For each experiment, the column was first wet-packed with the acid-clean sand prior to the transport study.

For the saturated transport experiments, the packed sand column was first flushed with DI water and electrolyte solution induced in a downward direction using a peristaltic pump (Masterflex LS, Cole Parmer Instrument, Vernon Hills, IL) at a rate of $1 \mathrm{~mL} \mathrm{~min}{ }^{-1}$. Hydrochemical equilibration was reached by applying 3 pore volumes (PVs) of DI water following 3 PVs of background solution made up of an electrolyte concentration (1, 10 or 100 $\mathrm{mM} \mathrm{NaCl}$ ) matching the $\mathrm{GO}$ suspension.

For the unsaturated transport experiments, two peristaltic pumps (Masterflex LS, Cole Parmer Instrument, Vernon Hills, IL) connected at the column inlet and outlet were used first to regulate the sand-pack moisture content and then to maintain downward and steady-state flow in the column. Initially, a saturated column was drained by elevating the outflow rate 5\% higher than the inflow rate. When the target moisture content was reached (i.e., 0.22), the inflow and outflow rates were equalized to $1 \mathrm{~mL} \mathrm{~min}^{-1}$. Similar to the procedure for saturated transport experiments, hydrochemical equilibration was achieved by pumping 3 PVs of DI water and then 3 PVs of background solution of an electrolyte concentration $(1,10$ or $100 \mathrm{mM} \mathrm{NaCl})$ matching the $\mathrm{GO}$ suspension.

The breakthrough experiment was then initiated by injecting at the column inlet a $3 \mathrm{PV}$ pulse 
of GO suspension (12 mg/L) at the above specified rates for each water saturation condition. The effluent of the column was sampled using a fraction collector every 4 minutes and the concentration of the GO effluent was monitored by UVS as described above. The same procedure for breakthrough experimentation and sample collection was used for both saturated and unsaturated experiments.

\subsection{Mathematical models}

The extended Derjaguin-Landau-Verwey-Overbeek (XDLVO) theory was used to calculate the interaction forces between GO particles, GO particles and quartz sand, and GO particles and the air-water interface. Here, interactions between GO particles control their stability in suspensions, interactions between GO and the sand are indicative of deposition in both saturated and unsaturated conditions, while interactions between GO and the sand as well as the air-water interface indicate the likelihood for deposition in unsaturated conditions. The XDLVO forces included van der Waals attraction, electric double layer repulsion, and Lewis acid-base $(\mathrm{AB})$ interactions. Details of the expression for each force are included in the Supporting Information (S1).

A transport model based on the advection-dispersion-reaction (ADR) theory was used to simulate the retention and transport of GO in the sand columns. The governing equations can be written as $[24,25]$ :

$\frac{\partial C}{\partial t}=D \frac{\partial^{2} C}{\partial z^{2}}-v \frac{\partial C}{\partial z}-\frac{\rho_{b}}{n} \frac{\partial S}{\partial t}$ 
$\frac{\partial S}{\partial t}=\frac{n}{\rho_{b}} k\left(1-\frac{S}{S_{\max }}\right) C$

where $C$ is the concentration of suspended GO in pore water $\left(\mathrm{M} \mathrm{L}^{-3}\right), t$ is time $(\mathrm{T}), D$ is the dispersion coefficient $\left(\mathrm{L}^{2} \mathrm{~T}^{-1}\right), z$ is the distance traveled in the direction of flow $(\mathrm{L}), v$ is the

average linear pore-water velocity $\left(\mathrm{L} \mathrm{T}^{-1}\right), \rho_{b}$ is the medium bulk density $\left(\mathrm{M} \mathrm{L}^{-3}\right), n$ is the porosity, $S$ is the deposited/retained particle concentration, $k$ is the particle retention constant, $S_{\max }$ is the maximum deposited/retained particle concentration. This model was applied to simulate the experimental breakthrough curves and was solved numerically with a finite-difference scheme for zero initial GO concentration, and pulse-input and zero-concentration-gradient boundary conditions at the column inlet and outlet, respectively.

\section{Results and discussion}

\subsection{XDLVO energy}

The zeta-potential values for GO particles and the quartz sand under the three ionic strength conditions are presented in Table 1 with which XDLVO forces were determined. Zeta-potential results indicate that the sand was consistently more negative than that of the GO particles under matching ionic strength condition. The XDLVO interaction energy profiles at the range of ionic strengths used are presented in Figure 1 for GO and GO (Figure 1a), GO and sand (Figure 1b), as well as for GO and the air-water interface (Figure 1c). Inserts illustrate secondary energy minimum wells. 
The XDLVO energy profiles between the GO particles (Figure 1a) show strong primary energy barriers higher than $7 \mathrm{~mJ} / \mathrm{m}^{2}$ (i.e., $1.7 \mathrm{kT} / \mathrm{nm}^{2}$ ) for all three ionic strength conditions, which is hard to overcome because the diameter of the GO particles was 1-5 $\mu \mathrm{m}$ (as reported by the manufacture). Thus, it can be concluded that, under the experimental conditions tested, aggregations of GO particles through primary minimum interactions were negligible. Previous studies indicated that increasing ionic strength conditions can reduce the energy barrier through reducing the repulsive electric double layer forces [26-28]. In this study, however, the primary energy barrier stayed almost unchanged when the solution ionic strength increase from $1 \mathrm{mM}$ to $10 \mathrm{mM}$ and $100 \mathrm{mM}$. It has been also reported in the literature that increasing ionic strength conditions may introduce secondary minima for nanoparticles to aggregate [27, 29-31]. The XDLVO energy profiles between the GO particles confirmed this and showed two secondary minimum wells at $-0.0013 \mathrm{~mJ} / \mathrm{m}^{2}\left(-0.00032 \mathrm{k} \mathrm{kT} / \mathrm{nm}^{2}\right)$ and $-0.021 \mathrm{~mJ} / \mathrm{m}^{2}\left(-0.0051 \mathrm{kT} / \mathrm{nm}^{2}\right)$ for the ionic strength of $10 \mathrm{mM}$ and $100 \mathrm{mM}$, respectively. These results suggested that the GO particles in the $10 \mathrm{mM}$ and $100 \mathrm{mM}$ electrolyte solutions could aggregate through the secondary minimum attachment.

Similarly, increasing ionic strength conditions had little effect on the primary energy barriers but deepened the secondary-minimum wells between GO and the sand surface (Figure 1b). The primary energy barriers of all three tested conditions were very high (around $7 \mathrm{~mJ} / \mathrm{m}^{2}$ ), suggesting these conditions were unfavorable for the attachment of the GO particles on the sand surfaces at the primary minima. However, the XDLVO energy profiles showed that the GO 
particles could deposit on the sand surfaces through the secondary minimum attachment at ionic strength of 10 and $100 \mathrm{mM}$.

The XDLVO energy profiles between the GO and the air-water interface (Figure 1c) were different from the above-mentioned profiles and showed much lower primary energy barriers. Increasing in ionic strength not only deepened the primary energy minimum wells (from -17 to $-25 \mathrm{~mJ} / \mathrm{m}^{2}$ ), but also reduce the primary energy barriers (from 0.16 to $0.03 \mathrm{~mJ} / \mathrm{m}^{2}$ ). The energy profiles of GO and the air-water interface did not exhibit secondary-energy minima for any condition, suggesting that the GO particles cannot attach to the air-water interfaces through the secondary minimum deposition.

\subsection{Stability of GO in different ionic strength conditions}

Temporal changes of concentrations of GO aqueous solutions under three different ionic strength conditions were exhibited in Figure 2. In general, the GO particles were stable in all three electrolyte solutions. The relative concentrations of GO particles in the suspension stayed unchanged under the $1 \mathrm{mM} \mathrm{NaCl}$ condition during the experiment, which is consistent with results of the XDLVO theory that the condition is unfavorable for the aggregation in neither primary nor secondary minima. When the ionic strength increased to $10 \mathrm{mM}$, it did not show obvious effect on the relative concentrations of the GO particles, although its XDLVO profile showed a secondary minimum at a separation distance at around $20 \mathrm{~nm}$. This inconsistency is probably because the secondary minimum well is relatively low. Under the $100 \mathrm{mM} \mathrm{NaCl}$ 
condition, the GO suspension became slightly less stable and the relative concentration reduced to about $0.95 \mathrm{C}_{0}$ (where $\mathrm{C}_{0}$ is the initial GO concentration) after $7 \mathrm{hrs}$. This result is consistent with the predictions of the XDLVO theory that high ionic strength may promote the aggregation of GO particles at the secondary minimum.

\subsection{GO transport in bubble column}

The changes of concentrations of GO with different temporal and spatial distributions in bubble columns are shown in Figure 3. The concentration of GO within the bubble column almost stayed unchanged for more than 4 hours with continuous bubble injections at the four locations. This result suggested that the experimental conditions are not favorable for the GO suspensions to deposit onto the air-water interfaces. This is probably because the GO particles could not overcome the energy barriers to reach the primary minimum wells, although the XDLVO energy profile showed deep primary minima for all three ionic strength conditions.

\subsection{Transport of GO in porous media}

Breakthrough curves of GO in saturated and unsaturated columns under different ionic strength conditions are shown in Figure 4. Under saturated conditions, the transport of GO exhibited high mobility with a recovery rate around $100 \%$ under the $1 \mathrm{mM} \mathrm{NaCl}$ Condition (Figure 4a). The retention of GO increased to $14.1 \%$ when the ionic strength was increased to $10 \mathrm{mM}$ (Table 2). The recovery rates of GO decreased dramatically when the ionic strength 
increased to $100 \mathrm{mM}$ and about $97 \%$ of the GO particles were retained in the column (Figure $4 \mathrm{a}$ ). These results are consistent with the XDLVO energy profiles of the interaction between GO particles and sand surfaces as shown in Figure 1b. Because the GO particles are stable under all the experimental ionic strength conditions (Figure 2), the retention of GO particles in the saturated porous media under higher ionic strength conditions can be mainly attributed to the deposition in the secondary minima. Other mechanisms, such as pore straining and ripening, may not be very important under the tested experimental conditions.

Ionic strength showed similar effect on GO transport in unsaturated porous media and the retention of GO particles in the unsaturated columns was around $4.4 \%, 24.6 \%$, and $98.5 \%$ for ionic strength of $1 \mathrm{mM}, 10 \mathrm{mM}$, and $100 \mathrm{mM}$, respectively (Table 2). This further confirmed the predictions of the XDLVO theory of the secondary minimum deposition under higher ionic strength conditions. Although the bubble column experiments indicated that air-water interface could not capture any GO particles for the three ionic strength conditions tested, the retention of GO in the unsaturated columns were about 3.8\%, $10.3 \%$ and $1.5 \%$ lower than that of saturated experiment, respectively. The enhanced retention of GO particles in the columns under unsaturated conditions could be caused by additional deposition mechanisms, such as film straining, which is commonly observed for colloid transport in unsaturated porous media [16, 17, $25,32]$. Additional investigations are still needed to further determine the mechanisms governing the deposition and transport of GO particles in unsaturated porous media.

We used the ADR model to simulate the experiments and the dispersion coefficient was 
obtained by the breakthrough data of bromide from our previous studies [23, 33-35]. The model simulations matched the experimental data very well for not only saturated sand column but also unsaturated condition. The $R^{2}$ and the best-fit values of the two fitting parameters (i.e., $k$ and $S_{\max }$ ) for GO were in Table 2. Although the maximum capacity term, $S_{\max }$, were used in all simulations, some of the experimental breakthrough curves did not reflect the influences of the previous depositions and the model simulations were insensitive to the $S_{\max }$. Thus, the reactive equation of the model (equation 2) could be reduced to the first-order kinetics equation by setting $S_{\max }$ to infinite for those simulations. For the ionic strength of $10 \mathrm{mM}$, the best-fit $S_{\max }$ value of GO transport in the unsaturated column was around $1.47 \mathrm{mg} \mathrm{g}^{-1}$, slightly higher than that in the saturated column $\left(1.28 \mathrm{mg} \mathrm{g}^{-1}\right)$. This results confirmed that addition deposition mechanism(s) could occur in unsaturated porous media to enhance the retention of GO particles. The best-fit $k$ values of GO transport under three ionic strength conditions ranged from 0.00018 to $0.12 \mathrm{~min}^{-1}$ and 0.0020 to $0.28 \mathrm{~min}^{-1}$ in saturated and unsaturated columns, respectively. As anticipated, the $k$ value increased as the water ionic strength increased for both saturated and unsaturated porous media. In addition, under the same ionic strength condition, both the best-fit parameter values and recovery rate of GO in unsaturated sand column were lower than that in saturated media. The results confirmed that GO has mobility in porous media, particularly high mobility under low ionic strength condition. Overall ionic strength played an important role in affecting the transport of GO in both saturated and unsaturated porous media. It can be anticipated that a mass of GO, when released into soils, might have a great impact on both the vadose zone and groundwater 
systems.

\section{Conclusions}

This study demonstrated that GO has high mobility in both saturated and unsaturated porous media under low ionic strength conditions. Increasing ionic strength could dramatically reduce the transport of GO particles in porous media. The result also suggested that moisture content also played an important role in the retention and transport of GO in porous media. Recovery rate of GO under unsaturated sand column were lower than which under saturated media in the same ionic strength condition. In addition, mathematic models based on the advection-dispersion-reaction equation could be used to simulate the retention and transport of GO in both saturated and unsaturated porous media. It can be concluded that a mass of GO, when released to groundwater systems, may have impacts on groundwater environment, as well as other aquatic systems.

\section{Acknowledgements}

This work was partially supported by the NSF through grant CBET-1054405, the NSFC through grant 51179203, and Donghua University through a scholarship to L. Liu. 


\section{References}

[1] H.A. Becerril, J. Mao, Z. Liu, R.M. Stoltenberg, Z. Bao, Y. Chen, Evaluation of Solution-Processed Reduced Graphene Oxide Films as Transparent Conductors, ACS Nano, 2 (2008) 463-470.

[2] D.A. Dikin, S. Stankovich, E.J. Zimney, R.D. Piner, G.H.B. Dommett, G. Evmenenko, S.T.

Nguyen, R.S. Ruoff, Preparation and characterization of graphene oxide paper, Nature, 2007.

[3] G. Eda, G. Fanchini, M. Chhowalla, Large-area ultrathin films of reduced graphene oxide as a transparent and flexible electronic material, Nature Publishing Group, 2008.

[4] B. Fugetsu, E. Sano, H. Yu, K. Mori, T. Tanaka, Graphene oxide as dyestuffs for the creation of electrically conductive fabrics, Carbon, 48 (2010) 3340-3345.

[5] K.A. Mkhoyan, A.W. Contryman, J. Silcox, D.A. Stewart, G. Eda, C. Mattevi, S. Miller, M. Chhowalla, Atomic and Electronic Structure of Graphene-Oxide, Nano Letters, 9 (2009) 1058-1063.

[6] D.R. Dreyer, S. Park, C.W. Bielawski, R.S. Ruoff, The chemistry of graphene oxide, Chemical Society Reviews, 39 (2010) 228-240.

[7] E.C. Salas, Z. Sun, A. Lüttge, J.M. Tour, Reduction of Graphene Oxide via Bacterial Respiration, ACS Nano, 4 (2010) 4852-4856.

[8] K. Wang, J. Ruan, H. Song, J. Zhang, Y. Wo, S. Guo, D. Cui, Biocompatibility of Graphene Oxide, Nanoscale Res. Lett., 6 (2011).

[9] O. Akhavan, E. Ghaderi, Toxicity of Graphene and Graphene Oxide Nanowalls Against 
Bacteria, ACS Nano, 4 (2010) 5731-5736.

[10] Y. Chang, S.-T. Yang, J.-H. Liu, E. Dong, Y. Wang, A. Cao, Y. Liu, H. Wang, In vitro toxicity evaluation of graphene oxide on A549 cells, Toxicology Letters, 200 (2011) 201-210.

[11] M. Hirata, T. Gotou, S. Horiuchi, M. Fujiwara, M. Ohba, Thin-film particles of graphite oxide 1:: High-yield synthesis and flexibility of the particles, Carbon, 42 (2004) 2929-2937. [12] G. Bin, X. Cao, Y. Dong, Y. Luo, L.Q. Ma, Colloid deposition and release in soils and their association with heavy metals, Crit Rev Env Sci Tec, 41 (2011) 336-372.

[13] M. Flury, H.X. Qiu, Modeling colloid-facilitated contaminant transport in the vadose zone, Vadose Zone J, 7 (2008) 682-697.

[14] L. Feriancikova, S. Xu, Deposition and remobilization of graphene oxide within saturated sand packs, Journal of Hazardous Materials, 235-236 (2012) 194-200.

[15] Y. Zevi, A. Dathe, B. Gao, W. Zhang, B.K. Richards, T.S. Steenhuis, Transport and retention of colloidal particles in partially saturated porous media: Effect of ionic strength, Water Resour Res, 45 (2009) W12403.

[16] B. Gao, T.S. Steenhuis, Y. Zevi, V.L. Morales, J.L. Nieber, B.K. Richards, J.F. McCarthy, J.Y. Parlange, Capillary retention of colloids in unsaturated porous media, Water Resour Res, 44 (2008) 7.

[17] B. Gao, J.E. Saiers, J. Ryan, Pore-scale mechanisms of colloid deposition and mobilization during steady and transient flow through unsaturated granular media, Water Resour Res, 42 (2006) W01410. 
[18] W.S. Hummers, R.E. Offeman, Preparation of Graphitic Oxide, J. Am. Chem. Soc., 80 (1958) 1339-1339.

[19] X. Rui, J. Zhu, D. Sim, C. Xu, Y. Zeng, H.H. Hng, T.M. Lim, Q. Yan, Reduced graphene oxide supported highly porous V2O5 spheres as a high-power cathode material for lithium ion batteries, Nanoscale, 3 (2011) 4752-4758.

[20] Y. Tian, B. Gao, L. Wu, R. Munoz-Carpena, Q. Huang, Effect of solution chemistry on multi-walled carbon nanotube deposition and mobilization in clean porous media, $\mathrm{J}$ Hazard Mater, 231 (2012) 79-87.

[21] P.R. Johnson, N. Sun, M. Elimelech, Colloid Transport in Geochemically Heterogeneous Porous Media: Modeling and Measurements, Environmental Science \& Technology, 30 (1996) 3284-3293.

[22] J. Wan, T.K. Tokunaga, Measuring Partition Coefficients of Colloids at Air-Water Interfaces, Environmental Science \& Technology, 32 (1998) 3293-3298.

[23] Y.A. Tian, B. Gao, K.J. Ziegler, High mobility of SDBS-dispersed single-walled carbon nanotubes in saturated and unsaturated porous media, J Hazard Mater, 186 (2011) 1766-1772.

[24] J. Smith, B. Gao, H. Funabashi, T.N. Tran, D. Luo, B.A. Ahner, T.S. Steenhuis, A.G. Hay, M.T. Walter, Pore-Scale Quantification of Colloid Transport in Saturated Porous Media, Environmental Science \& Technology, 42 (2007) 517-523.

[25] B. Gao, J.E. Saiers, J.N. Ryan, Deposition and mobilization of clay colloids in unsaturated porous media, Water Resour Res, 40 (2004) W08602. 
[26] W.P. Johnson, M. Tong, X. Li, On colloid retention in saturated porous media in the presence of energy barriers: The failure of alpha, and opportunities to predict eta, Water Resour Res, 43 (2007) -.

[27] J.A. Redman, S.L. Walker, M. Elimelech, Bacterial adhesion and transport in porous media: Role of the secondary energy minimum, Environ Sci Technol, 38 (2004) 1777-1785.

[28] M.W. Hahn, C.R. O'Melia, Deposition and reentrainment of Brownian particles in porous media under unfavorable chemical conditions: Some concepts and applications, Environ Sci Technol, 38 (2004) 210-220.

[29] N. Tufenkji, M. Elimelech, Breakdown of colloid filtration theory: Role of the secondary energy minimum and surface charge heterogeneities, Langmuir, 21 (2005) 841-852.

[30] M.W. Hahn, D. Abadzic, C.R. O'Melia, Aquasols: On the role of secondary minima, Environ Sci Technol, 38 (2004) 5915-5924.

[31] C.Y. Shen, B.G. Li, Y.F. Huang, Y. Jin, Kinetics of coupled primary- and secondary-minimum deposition of colloids under unfavorable chemical conditions, Environ Sci Technol, 41 (2007) 6976-6982.

[32] Y. Zevi, A. Dathe, B. Gao, B.K. Richards, T.S. Steenhuis, Quantifying colloid retention in partially saturated porous media, Water Resour Res, 42 (2006) 13.

[33] Y. Tian, B. Gao, Y. Wang, V.L. Morales, R.M. Carpena, Q.G. Huang, L.Y. Yang, Deposition and transport of functionalized carbon nanotubes in water-saturated sand columns, J Hazard Mater, 213 (2012) 265-272. 
[34] H.M. Sun, B. Gao, Y.A. Tian, X.Q. Yin, C.R. Yu, Y.Q. Wang, L.N.Q. Ma, Kaolinite and lead in saturated porous media: facilitated and impeded transport, J Environ Eng-asce, 136 (2010) 1305-1308.

[35] Y. Tian, B. Gao, V. Morales, Y. Wang, L. Wu, Effect of surface modification on single-walled carbon nanotube retention and transport in saturated and unsaturated porous media, J Hazard Mater, 239-240 (2012) 333-339. 


\section{Figure Captions}

Figure 1. XDLVO energy between GO particles (a), GO and sand (b), and GO and air-water interface under three different ionic strength conditions.

Figure 2. Stability of GO in aqueous solutions under three different ionic strength conditions.

Figure 3. GO concentrations at four sampling ports in the bubble columns measured at different time intervals and ionic strength conditions.

Figure 4. Transport of GO in saturated (a) and unsaturated (b) sand columns under different ionic strength conditions. Symbols are experimental data and lines are model results (simulated data). 


\section{Tables}

Table 1. Surface properties of the GO and sand used in this work

\begin{tabular}{lcclllll}
\hline & \multicolumn{3}{c}{ Electrophoretic mobility $\left(* 10^{-8} \mathrm{~m}^{2} / \mathrm{Vs}\right)$} & \multicolumn{3}{c}{ Zeta-potential $(\mathrm{mV})$} \\
& $1 \mathrm{mM}$ & $10 \mathrm{mM}$ & $100 \mathrm{mM}$ & $1 \mathrm{mM}$ & $10 \mathrm{mM}$ & $100 \mathrm{mM}$ \\
\hline GO & -2.93 & -2.7 & -2.24 & -37.48 & -34.42 & -28.64 \\
Sand & -4.99 & -4.25 & -2.65 & -63.82 & -54.37 & -33.9 \\
\hline
\end{tabular}

Table 2. Best-fit parameter values and recovery rate calculation for GO in saturated $(0.40)$ and unsaturated $(0.22)$ porous media.

\begin{tabular}{llllll}
\hline Moisture content & Ionic strength & $\mathrm{S}_{\max }\left(\mathrm{mg} \mathrm{g}^{-1}\right)$ & $\mathrm{K}\left(\mathrm{min}^{-1}\right)$ & Recovery (\%) & $\mathrm{R}^{2}$ \\
\hline 0.40 & $1 \mathrm{mM}$ & - & $1.80 \mathrm{E}-04$ & 99.4 & 0.99 \\
0.40 & $10 \mathrm{mM}$ & 1.28 & $6.74 \mathrm{E}-03$ & 85.9 & 0.99 \\
0.40 & $100 \mathrm{mM}$ & - & $1.18 \mathrm{E}-01$ & 3.0 & 0.55 \\
0.22 & $1 \mathrm{mM}$ & - & $2.00 \mathrm{E}-03$ & 95.6 & 0.99 \\
0.22 & $10 \mathrm{mM}$ & 1.47 & $3.07 \mathrm{E}-02$ & 75.6 & 0.99 \\
0.22 & $100 \mathrm{mM}$ & - & $2.79 \mathrm{E}-01$ & 1.5 & 0.88 \\
\hline
\end{tabular}


Figure 1
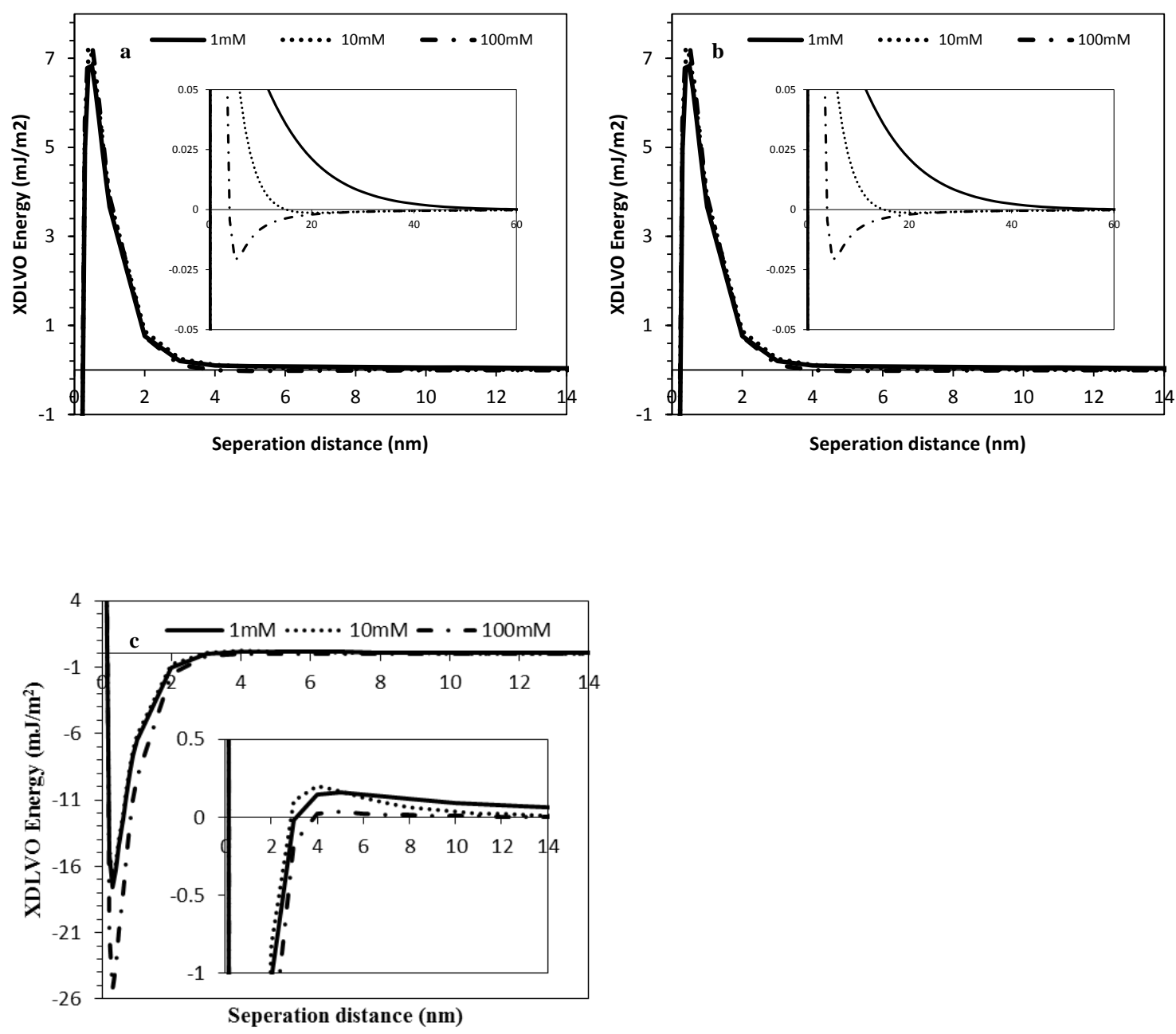
Figure 2

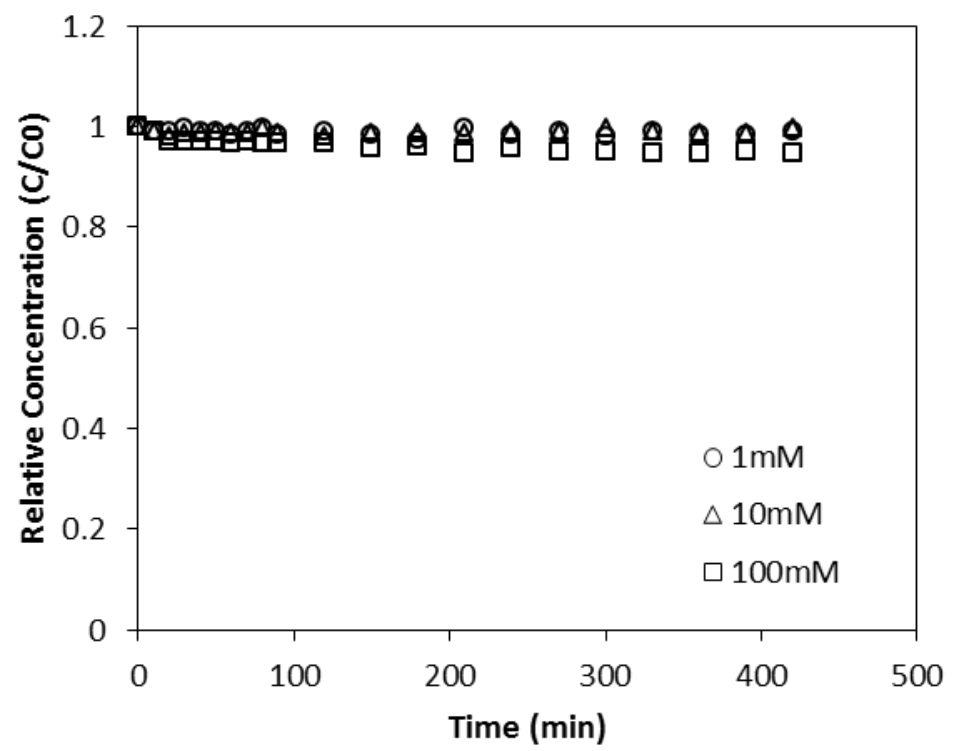


Figure 3

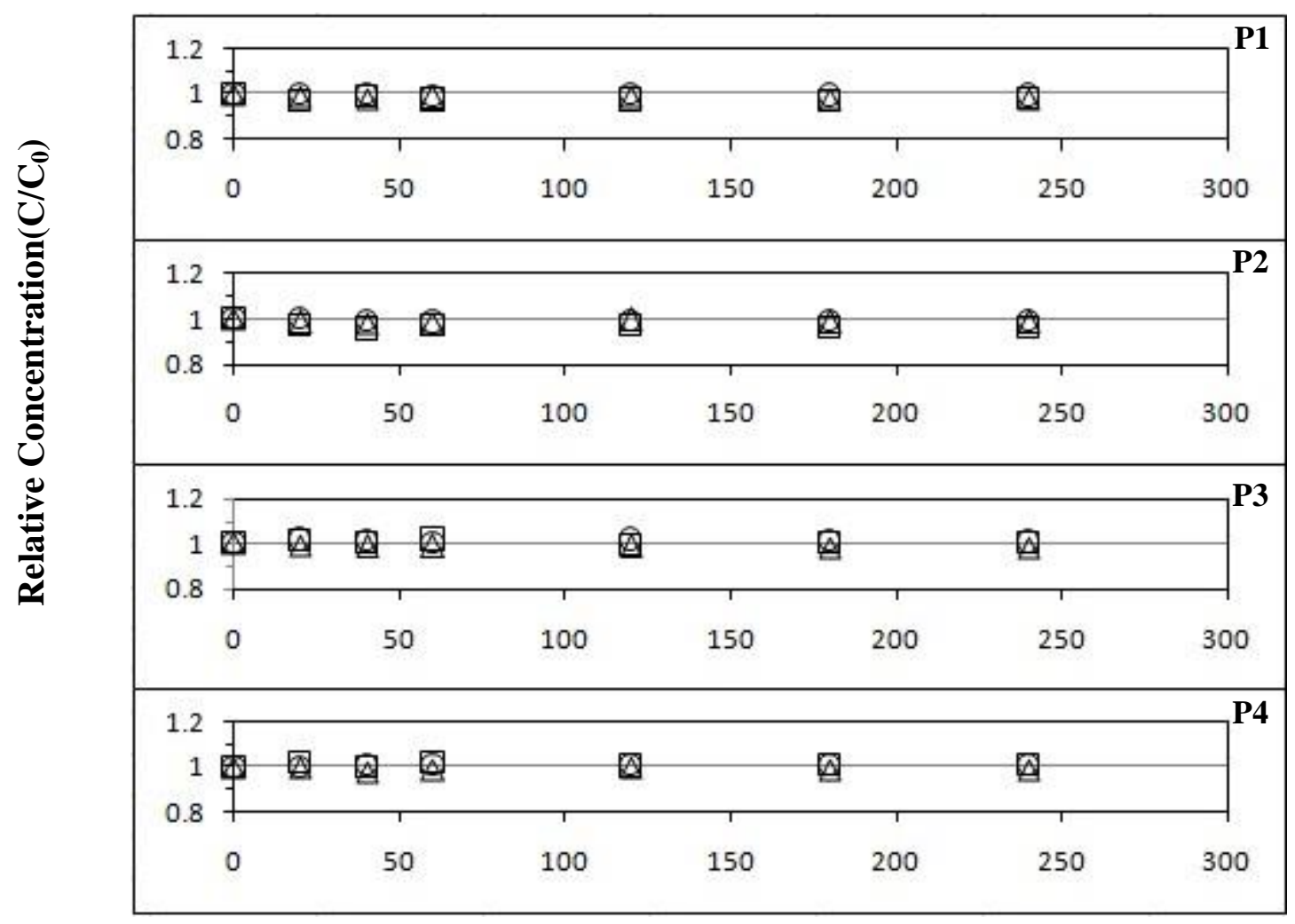

$01 \mathrm{~mm}$

$\triangle 10 \mathrm{mM}$

$\square 100 \mathrm{mM}$

Time (min) 
Figure 4
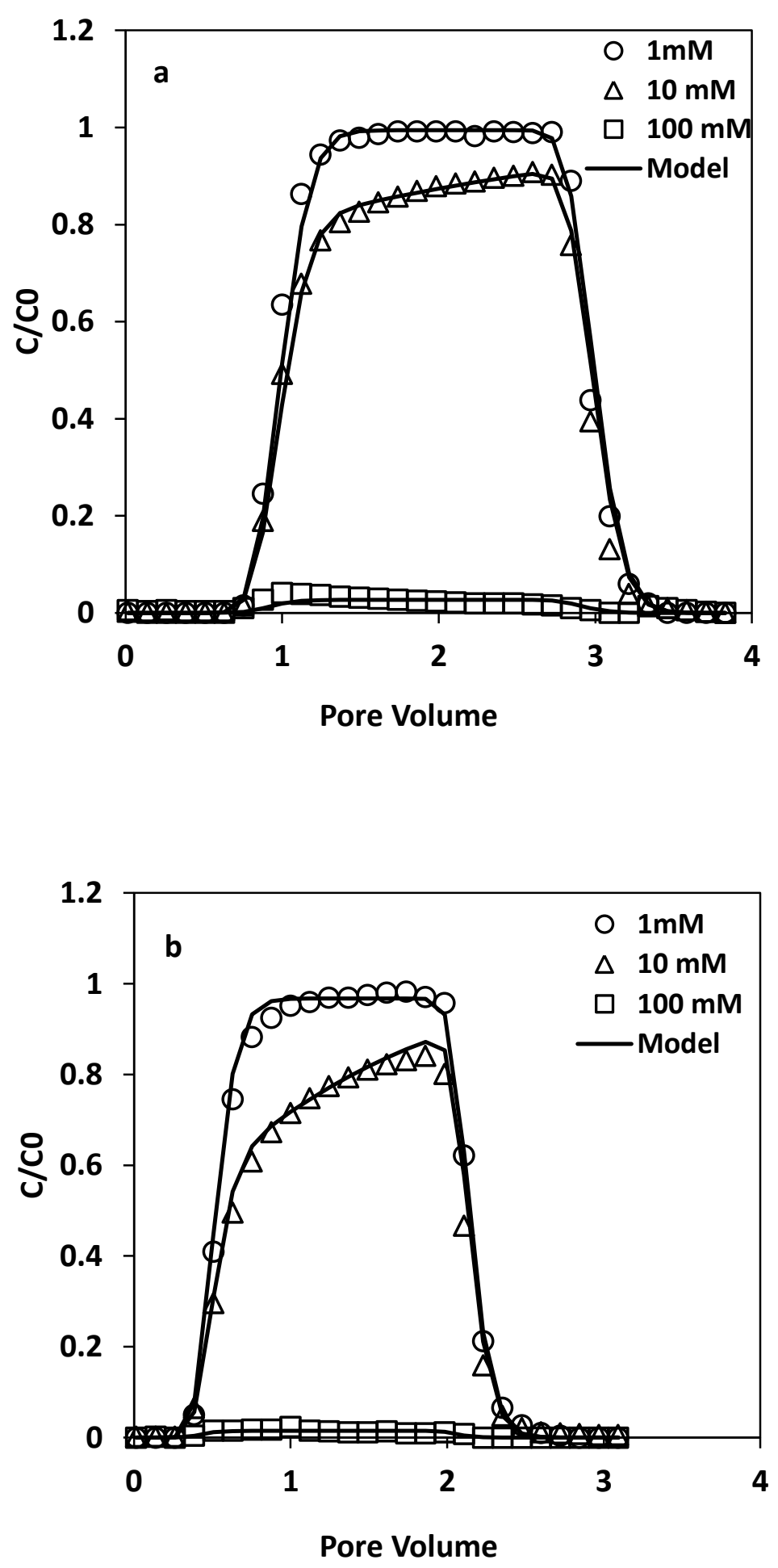